Paula Cohen and J. Wolfart, Modular embeddings for some non-arithmetic Fuchsian groups . . . . . . . . . . . . . . . . . . Noriko Hirata-Kohno, Mesures de transcendance pour les quotients de périodes d'intégrales elliptiques . . . . . . . . . . . . . . . . . . 111-133

A. Ivić, Large values of certain number-theoretic error terms . . . . . . . . . 135-159

W. M. Schmidt, Eisenstein's theorem on power series expansions of algebraic functions . . . . . . . . . . . . . . . . . . . . . . . . 161-179

A. Schinzel, Addendum to the paper "On two theorems of Gelfond and some of their applications" (Acta Arith. 13 (1967), pp. 177-236) . . . . . . . . . . .

\title{
Modular embeddings for some non-arithmetic Fuchsian groups
}

by

\author{
Paula Cohen (Paris) and Jürgen Wolfart (Frankfurt)
}

La revue est consacrée à la Théorie des Nombres

The journal publishes papers on the Theory of Numbers

Die Zeitschrift veröffentlicht Arbeiten aus der Zahlentheorie

Журнал посвящен теории чисел

\section{$\begin{array}{lll}\text { Ladresse de } & \begin{array}{l}\text { Address of the } \\ \text { Editorial Board }\end{array} & \begin{array}{l}\text { Die Adresse der } \\ \text { Schriftleitung }\end{array}\end{array}$ \\ ACTA ARITHMETICA}

ul. Sniadeckich 8, skr. poczt. 137, 00-950 Warszawa, telex PL 816112

Les auteurs sont priés d'envoyer leurs manuscrits en deux exemplaires à l'adresse ci-dessus The authors are requested to submit papers in two copies to the above address

Die Autoren sind gebeten um Zusendung von 2 Exemplaren jeder Arbeit an die obige Adresse Рукописи статей редакция просит присылать в двух экземплярах по вышеуказанному адрессу

\begin{tabular}{|c|c|c|c|}
\hline $\begin{array}{l}\text { Abonnements } \\
\text { Échange } \\
\text { Numerós antérieurs }\end{array}$ & $\begin{array}{l}\text { Subscriptions } \\
\text { Library exchange } \\
\text { Back numbers }\end{array}$ & $\begin{array}{l}\text { Abonnement } \\
\text { Austausch } \\
\text { Ankauf }\end{array}$ & $\begin{array}{l}\text { Подписка } \\
\text { Книгообмен } \\
\text { Продажа }\end{array}$ \\
\hline
\end{tabular}

Instytut Matematyczny Polskiej Akademii Nauk skr. poczt. 137, 00-950 Warszawa, telex PL 816112

(C) Copyright by Instytut Matematyczny PAN, Warszawa 1990

Published by PWN-Polish Scientific Publishers

ISBN 83-01-09918-6 ISSN 0065-1036

PRINTED IN POLAND
0. Introduction. The purpose of the present paper is to shed further light on results of [Wo 1], [Wo 2], [WW] which concern the following transcendence question in uniformization theory, first raised by S. Lang [La]:

Let $X$ be a smooth projective algebraic curve of genus $g>1$, defined over the field $\bar{Q}$ of algebraic numbers. Suppose that the universal holomorphic covering map

$$
\varphi: E_{\varrho}:=\{z \in C|| z \mid<\varrho\} \rightarrow X
$$

is normalized in such a way that $x_{0}=\varphi(0) \in X(\bar{Q})$ is an algebraic point of $X$ and the tangent map $\varphi^{\prime}(0)$ is defined over $\bar{Q}$ : is the covering radius $\varrho$ then a transcendental number?

Up to non-zero algebraic factors, $\varrho$ is uniquely determined as a function of $X, x_{0}$, by the above normalization. Of course, it still makes sense to ask Lang's question for covering maps with finitely many possibly ramified points of $X(\bar{Q})$ removed.

The transcendence of $\varrho$ can be established in the following cases:

1) $X$ a modular curve, $x_{0}$ a complex multiplication point (henceforth referred to as a C-M point). This uses work of C. Siegel [Sie 2] and is the subject of [Wo2].

2) $X$ a Shimura curve, $x_{0}$ a C-M point. This falls into the context of work of G. Shimura [Shi 2], and is elaborated on in our Section 5.

3) $X$ a curve with covering group of finite index in a Fuchsian triangle group having $x_{0}$ as a non-trivial fixed point. Such curves with many automorphisms are discussed in [Wo 3], [WW] and our Sections 1 and 4.

Moreover, in each of the above cases, the covering radius turns out to be a quotient of a period of the first kind by a period of the second kind on certain abelian varieties with complex multiplication. The transcendence of such quotients follows from theorems of $\mathrm{G}$. Wüstholz [WW, §1]. However, in contrast to cases 1 and 2, the occurrence in case 3 of these abelian varieties is a priori somewhat strange, since in general the covering group $\Delta$ of $X$ is not arithmetically defined.

This paper focuses precisely on elucidating this last point: we shall prove

.


that there is a $\bar{Q}$-rational morphism $\psi$ of $X$ into a suitable Shimura variety $V$ which maps $x_{0}$ to a C-M point of $V$. This gives a reason, consistent with 1) and 2), for the dependence in 3) of $\varrho$ on periods of abelian varieties with complex multiplication. The mapping $\psi$ is induced by an embedding of $\Delta$ into the modular group $\Gamma$ associated to $V$, hence the description "modular embedding" in the title, together with an analytic embedding of the covering space of $X$ into that of $V$, compatible with the action of $\Delta$.

It appears that such modular embeddings have previously been considered only for arithmetic groups. The name was first used in a paper of Hammond [Ha], but the idea is older, see for example [Kli] or [Fr]. Modular embeddings for some non-arithmetic groups implicitly occur in [Wo 3]. We hope that the expanded list of examples treated in this paper has applications beyond the context of Lang's question: in particular, in the spirit of Shimura's work, it may make available useful models for some deeper arithmetic issues.

The paper is organized as follows: after the preliminaries of Section 1, Section 2 introduces the main result about modular embeddings, various analytic proofs of which are provided in Section 3, the $\bar{Q}$-rationality of the morphism $\psi$ being settled in Section 4. Next, Section 5 reviews the consequences for the covering radius, Section 6 gives some examples and Section 7 offers suggestions for extending the ideas of the present paper to a wider class of Fuchsian groups.

1. Preliminaries on triangle and modular groups. Let $p, q, t$ be positive integers satisfying $1 / p+1 / q+1 / t<1$, with $q, t$ possibly infinite. We define a Fuchsian triangle group $\Delta \subset \mathrm{SL}_{2}(R)$ of signature $(p, q, t)$ to be any group generated by matrices

$$
\gamma_{P}, \gamma_{Q}, \gamma_{T},-1_{2}=\left[\begin{array}{rr}
-1 & 0 \\
0 & -1
\end{array}\right] \in \mathrm{SL}_{2}(\boldsymbol{R})
$$

satisfying

$$
\text { (2) } \operatorname{tr} \gamma_{P}= \pm 2 \cos (\pi / p), \quad \operatorname{tr} \gamma_{Q}= \pm 2 \cos (\pi / q), \quad \operatorname{tr} \gamma_{T}= \pm 2 \cos (\pi / t) \text {. }
$$

One may realize the images $\bar{\gamma}_{P}, \bar{\gamma}_{Q}, \bar{\gamma}_{T}, 1$ of these generators in $\operatorname{PSL}_{2}(\boldsymbol{R})$ as fractional linear transformations acting on the upper half plane $\mathfrak{h}$. Then $\bar{\gamma}_{P}, \bar{\gamma}_{Q}, \bar{\gamma}_{T}$ have fixed points $P, Q, T$ forming the vertices of a closed hyperbolic triangle $F_{0}$, with angles $\pi \lambda=\pi / p, \pi \mu=\pi / q, \pi \nu=\pi / t$. One may suppose $P, Q$, $T$ all lie in $\mathfrak{h}$, or on the boundary $\boldsymbol{R} \cup\{\infty\}$ if the corresponding angles are 0 . Let $F_{0}^{\prime}$ be the triangle with vertices $P Q^{\prime} T$, image of $F_{0}$ under reflexion in the side $P T$. Geometrically, $\bar{\gamma}_{P}, \bar{\gamma}_{Q}, \bar{\gamma}_{T}$ are hyperbolic rotations with rotation angle $2 \pi / p$, $2 \pi / q, 2 \pi / t$. If $Q$ or $T$ is realized as a cusp on $\partial \mathfrak{h}$, the corresponding trace becomes \pm 2 and $\gamma_{Q}$ or $\gamma_{T}$ becomes a parabolic transformation. Since hyperbolic triangles are uniquely determined by their angles up to hyperbolic motions, $\Delta$ is uniquely determined by its signature $(p, q, t)$ up to conjugation in $\mathrm{SL}_{2}(R)$ and a possible extension by $-1_{2}$.

Notice that the signature $(\infty, \infty, \infty)$ is excluded only for practical reasons; in this case $\Delta$ is $\mathrm{SL}_{2}(R)$ conjugate to the principal congruence subgroup $\Gamma(2)$ of the elliptic modular group, and the interpretation of the covering radius by means of a modular embedding is immediate. We remark in passing that one is led to consider euclidean triangle groups if $1 / p+1 / q+1 / t=1$ and spherical triangle groups if $1 / p+1 / q+1 / t>1$.

Returning to the hyperbolic case, standard geometric arguments show that in the above discussion $P, Q, T$ can be chosen as algebraic numbers. In particular, $\Delta$ is $\mathrm{SL}_{2}(R)$ conjugate to a subgroup of $\mathrm{SL}_{2}(\boldsymbol{k})$ where $\boldsymbol{k}=\boldsymbol{k}_{4}$ is the totally real number field $Q(\cos (\pi / p), \cos (\pi / q), \cos (\pi / t))$.

The field $\boldsymbol{k}$ is crucial in what follows. Results of Takeuchi [Ta 1, Props. $2,3]$ show that its ring of integers contains the traces of all elements of $\Delta$, and that in the algebra $M_{2}(R)$ of $2 \times 2$ matrices over the reals, any subgroup $\Delta_{0}$ of finite index in $\Delta$ generates an order $\mathcal{O}$ of a quaternion algebra $\mathfrak{B}$ defined over its trace field $\boldsymbol{k}_{0}=\boldsymbol{Q}\left(\operatorname{tr} \Delta_{0}\right) \subset \boldsymbol{k}, \operatorname{tr} \Delta_{0}=\left\{\operatorname{tr} \gamma \mid \gamma \in \Delta_{0}\right\}$. Realizing the quaternionic reduced norm of $\mathfrak{B}$ as the matrix determinant, we can consider $\Delta_{0}$ as a subgroup of the group $\Gamma$ of units of $\mathcal{U}$ with reduced norm 1 .

General facts about quaternion algebras ensure the existence of an $\boldsymbol{R}$-algebra isomorphism

$$
\text { l: } \mathfrak{B} \otimes_{\mathbf{Q}} \boldsymbol{R} \rightarrow A=: M_{\mathbf{2}}(\boldsymbol{R}) \oplus \ldots \oplus M_{\mathbf{2}}(\boldsymbol{R}) \oplus \boldsymbol{H} \oplus \ldots \oplus \boldsymbol{H}
$$

where $A$ comprises $r$ copies of $M_{2}(R)$, and $n-r$ copies of the hamiltonians $\boldsymbol{H}$, with componentwise algebraic operations, and $n=\left[\boldsymbol{k}_{0}: Q\right]$. If $k_{j}$ denotes the projection of $A$ onto the $j$ th summand, then $\sigma_{j}=k_{j} \mathrm{Ol}$ is an embedding of $\mathfrak{B}$ in $M_{2}(R)$ for $j=1, \ldots, r$ and in $\boldsymbol{H}$ for $j=r+1, \ldots, n$ extending the $n$ field embeddings of $\boldsymbol{k}_{0}$ into $\boldsymbol{R}$. We can assume $\sigma_{1}$ is the identity map and write $\gamma_{j}=\sigma_{j}(\gamma), \gamma \in \mathfrak{B}$. There is an induced action of $\Gamma$ on $\mathfrak{h}^{r}$ given by

$$
\gamma: \tau=\left(\tau_{1}, \ldots, \tau_{r}\right) \mapsto\left(\gamma_{1}\left(\tau_{1}\right), \ldots, \gamma_{r}\left(\tau_{r}\right)\right)
$$

where $\gamma_{j}\left(\tau_{j}\right)$ denotes the fractional linear transformation of $\tau_{j}$ by $\gamma_{j}, j=1, \ldots, r$.

This action of $\Gamma$ is discontinuous, and $\Gamma$ is even a modular group for a suitably chosen family of polarized abelian varieties [Shi 1,2]. Indeed, let $\boldsymbol{K}$ be an imaginary quadratic extension of $\boldsymbol{k}_{0}$ contained in $\mathfrak{B}$ and extend the embedding $\sigma_{j}: \boldsymbol{k}_{0} \rightarrow \boldsymbol{R}$ to an embedding $\xi_{j}: \boldsymbol{K} \rightarrow \boldsymbol{C}$ and its complex conjugate $\xi_{j}, j=1, \ldots, r$. Then by work of Albert [A], Siegel [Sie 1] and Shimura [Shi 1] there is a family of principally polarized abelian varieties $\mathfrak{I}$, parametrized by $\tau \in \mathfrak{h}^{r}$, of complex dimension $2 n$ and with endomorphism algebra

$$
\text { End }_{0} \mathfrak{I}=\boldsymbol{Q} \otimes \text { End } \mathfrak{I} \supset \boldsymbol{K}
$$


and generalized complex multiplication type

$$
\phi=\sum_{j=1}^{r}\left(\xi_{j}+\bar{\xi}_{j}\right)+2 \sum_{j=r+1}^{n} \xi_{j} .
$$

The type $\phi$ encodes the induced action of $\boldsymbol{K}$ on the complex vector space of holomorphic 1 -forms of $\mathfrak{T}$. This action determines $2 r$ one-dimensional eigenspaces on which $\xi_{j} \boldsymbol{K}$ and $\bar{\xi}_{j} \boldsymbol{K}$ act by scalar multiplication, $j=1, \ldots, r$, and $n-r$ two-dimensional eigenspaces on which $\xi_{r+1} K, \ldots, \xi_{n} K$ act by scalar multiplication. Two members of this family of abelian varieties are isomorphic exactly when their corresponding parameters in $\mathfrak{h}^{r}$ lie in the same orbit of $\Gamma$, or at worst a group commensurable with $\Gamma$, if for example $\mathcal{O}$ is not a maximal order. The orbit space $\Gamma \backslash \mathfrak{h}^{r}$ therefore parametrizes the isomorphism classes of the abelian varieties in the family, and can itself be equipped with the structure of a complex space. Suitably chosen $\Gamma$-automorphic forms define a holomorphic embedding of $\Gamma \backslash \mathrm{h}^{r}$ into a complex projective space. If $\Gamma \backslash \mathrm{h}^{r}$ is not compact, which can only be the case if $r=n$, this embedding extends in a natural way to the cusps of $\Gamma$ and the compactification $\overline{\Gamma \backslash \mathfrak{b}^{r}}$ may be identified with a complex projective variety $V$, the so-called Shimura variety associated to $\Gamma$. Moreover, the embedding can be chosen in such a way that $V$ is defined over $\bar{Q}$.

2. The main result. We are now ready to formulate the main result of this paper:

THEOREM. Let $\Delta_{0}$ be a subgroup of finite index in a Fuchsian triangle group $\Delta$, and $X=\overline{\Delta_{0} \backslash \mathfrak{h}}$ the corresponding algebraic curve. Let $\Gamma$ be the norm-unit group of the quaternion algebra $\mathfrak{B}=\boldsymbol{k}_{0}\left[\Delta_{0}\right]$, where $\boldsymbol{k}_{0}$ is the trace field of $\Delta_{0}$ and $V=\overline{\Gamma \backslash \mathfrak{b}^{r}}$ its Shimura variety. Then there is an injective, non-singular, complex analytic embedding, $F: \mathfrak{h} \rightarrow \mathfrak{h}^{r}$, where $r$ divides the number of unramified infinite places of $\boldsymbol{k}$ for $\mathfrak{B}$, compatible with the inclusion of groups $l: \Delta_{0} \subseteq \Gamma$, so that

$$
F(\gamma(\tau))=\imath(\gamma) F(\tau), \quad \tau \in \boldsymbol{h}, \gamma \in \Delta_{0} .
$$

Moreover, there is a continuous extension of $F$ to include the cusps of $\Delta_{0}$, whose images are again cusps of $\Gamma$. Finally, $F$ induces on $X$ a $\bar{Q}$-rational morphism

$$
\psi: X \rightarrow V \text {. }
$$

If in addition $\imath\left(\Delta_{0}\right)=\{\gamma \in \Gamma \mid \gamma F(\mathfrak{h})=F(\mathfrak{b})\}$ the quotient map $\psi$ is a $\bar{Q}$-rational immersion. For example this condition holds if $\Delta_{0}=\Delta$ is a maximal triangle group, or in certain cases by replacing $\Gamma$ by a subgroup of finite index, a process which fails in general as, for example, when $\Gamma$ has only congruence subgroups but $\Delta_{0}$ has non-congruence subgroups.

Notice that the new contribution of the theorem is that it holds for non-arithmetic triangle groups. Indeed, $\Delta$ is arithmetic exactly when one may choose $r=1$ in the theorem: K. Takeuchi [Ta 1] has shown that this is possible for 85 signatures. Then, $X=V$ is a Shimura curve, or a modular curve if $\Delta$ has cusps and thus is commensurable to $\mathrm{SL}_{2}(Z)$, so that the existence of a modular embedding is immediate.

3. Construction of the modular embedding. By standard arguments, it suffices to prove the Theorem of Section 2 in the case where $\Delta=\Delta_{0}$ is a maximal triangle group (in particular, see [WW, Props. 4, 5]). We have to construct a complex analytic mapping:

$$
\begin{aligned}
F=\left(f_{1}, \ldots, f_{r}\right): \mathfrak{h} & \rightarrow \mathfrak{h}^{r} \\
\tau & \mapsto\left(f_{1}(\tau), \ldots, f_{r}(\tau)\right)
\end{aligned}
$$

satisfying for all $\tau$ in $\mathfrak{h}$, and $\gamma$ in $\Delta$

$$
f_{j}(\gamma \tau)=\gamma_{j} f_{j}(\tau), \quad j=1, \ldots, r .
$$

Since $\gamma_{1}=\gamma, f_{1}$ commutes with $\Delta$ and therefore maps every corner of the associated triangle onto itself, so by Schwarz' lemma $f_{1}$ is the identity map. This leaves us with the construction of $f_{2}, \ldots, f_{r}$. We shall present here three different but related methods of construction.

1. Geometric construction. Fix a $j$ between 2 and $r$ and denote $\sigma_{j}(\Delta)$ by $\Delta^{\sigma}$ and $\sigma_{j}(\gamma)=\gamma_{j}$ by $\gamma^{\sigma}$. The normalization of $\Delta$ chosen in Section 1 dictates that $\sigma_{j}$ act on the $\gamma^{\prime}$ 's by Galois conjugation of their matrix entries. In particular, the image of each generator $\gamma_{P}, \gamma_{Q}, \gamma_{T}$ of $\Delta$ is a generator $\gamma_{P}^{\sigma}, \gamma_{Q}^{\sigma}$ or $\gamma_{T}^{\sigma}$ of $\Delta^{\sigma}$, the ellipticity of a given order, or parabolicity, being respected. Thereby,

$$
\operatorname{tr} \gamma_{P}^{\sigma}= \pm 2 \cos (\pi m / p), \quad \operatorname{tr} \gamma_{Q}^{\sigma}= \pm 2 \cos (\pi s / q), \quad \operatorname{tr} \gamma_{T}^{\sigma}= \pm 2 \cos (\pi u / t),
$$

with $0<m<p, 0<s<q, 0<u<t$ satisfying $(m, 2 p)=(s, 2 q)=(u, 2 t)=1$. In this way, the fixed points $P^{\sigma}, Q^{\sigma}, T^{\sigma}$ of $\gamma_{P}^{\sigma}, \gamma_{Q}^{\sigma}, \gamma_{T}^{\sigma}$ in $h$ or on its boundary define a hyperbolic triangle of the same type as before, but with angles enlarged by integral multiples.

The hyperbolic triangle is a simply connected region whose boundary is analytic except at its three vertices. Therefore, by Riemann's mapping theorem with boundary, there is a bi-holomorphic mapping $f$ of the interior of the hyperbolic triangle $F_{0}=P Q T$ to the interior of the hyperbolic triangle $F_{0}^{\sigma}=P^{\sigma} Q^{\sigma} T^{\sigma}$ which is holomorphically extendable to the sides and continuously extendable to the vertices of the triangle where:

$$
f(P)=P^{\sigma}, \quad f(Q)=Q^{\sigma}, \quad f(T)=T^{\sigma} .
$$

Suppose we reflect $F_{0}$ in the hyperbolic side $P T$ as in Section 1 to obtain a second triangle $F_{0}^{\prime}$. Then $\Re=F_{0} \cup F_{0}^{\prime}$ is a fundamental region for $\Delta$. The boundary points of $\Re$ are congruent in pairs under $\Delta$ : in fact $\gamma_{P}(P)=P$, $\gamma_{P}(Q)=Q^{\prime}, \gamma_{T}(T)=T, \gamma_{T}(Q)=Q^{\prime}$. Moreover each of the three elliptic transfor- 
mations $\gamma_{P}, \gamma_{Q}, \gamma_{T}$, which generate $\Delta$, is a product of two hyperbolic reflections in sides of the triangle $F_{0}$. The function $f$ can be analytically continued across the open arc $P T$ in such a way as to map points which are conjugate under reflection in $P T$ to points which are conjugate under reflection in $P^{\sigma} T^{\sigma}$. One therefore has a corresponding region $\Re^{\sigma}=F_{0}^{\sigma} \cup F_{0}^{\sigma^{\prime}}$ where $F_{0}^{\sigma^{\prime}}=f\left(F_{0}^{\prime}\right)$ is the image of $F_{0}^{\sigma}$ under reflection in the line $P^{\sigma} T^{\sigma}$. The images of $\Re$ under the discrete group $\Delta$ cover $\mathfrak{b}$ by a system of non-overlapping hyperbolic triangles fitting together without lacunae. Therefore, repeating the principle for reflection in the hyperbolic side $P T$ (Schwarz' reflection principle) successively to all images under $\Delta$ of the sides of the fundamental domain $\mathfrak{R}$, one obtains a continuation of $f$ to the universal cover of $\mathfrak{h}^{\prime}=\mathfrak{h}-\Delta(\{P, Q, T\})$. This continuation gives a well-defined analytic function on $\mathfrak{h}^{\prime}$ itself, since the compatibility of the values of $f$ around a given element of the orbit of $P, Q$ $T$ under $\Delta$ is assured by the fact that the angles of $F_{0}^{\sigma}$ at $P^{\sigma}, Q^{\sigma}, T^{\sigma}$ are respectively multiples by $m, s, u$ of the angles of $F_{0}$ at $P, Q, T$. Moreover, due to the fact that every element of $\Delta$ is a product of hyperbolic reflections in appropriate sides of images of $\Re$ under $\Delta$, the reflection principle also shows that $f \circ \gamma=\gamma^{\sigma} \circ f$ for all $\gamma \in \Delta$.

To complete the continuation of $f$, we observe that $f$ is holomorphically extendable to the images in $\mathfrak{h}$ of $P, Q, T$ under $\Delta$, as these are removable singularities, giving values respectively of multiplicity $m, s, u$ : cusps, if any, are applied continuously in the appropriate topology to the parabolic fixed points. The resulting function is the $f_{j}$ required, since we clearly have:

$$
f_{j}(\gamma(\tau))=\gamma^{\sigma} f_{j}(\tau) \quad \text { for } \gamma \in \Delta, \tau \in \mathbf{b} .
$$

2. Construction using linear differential equation. Again fixing $j$ between 2 and $r$, the groups $\Delta$ and $\Delta^{\sigma}=\Delta_{j}$, as we have introduced them above, are explicit realizations of monodromy groups of linear differential equations

$$
L(w)=0 \quad \text { and } \quad L_{j}(w)=0
$$

of second order with coefficients in $\boldsymbol{C}(z)$ and regular singularities at $0,1, \infty$. They are hypergeometric differential equations, that of $\Delta$ being for the parameters

$$
a=\frac{1}{2}(1-\lambda-\mu-v), \quad b=\frac{1}{2}(1-\lambda+\mu-v), \quad c=1-\lambda
$$

and that of $\Delta_{j}$ being for the parameters $a_{j}, b_{j}, c_{j}$ obtained from $a, b, c$ by replacing $\lambda$ by $m \lambda, \mu$ by $s \mu$, and $\nu$ by $u v$. Both $L$ and $L_{j}$ have two linearly independent solutions each, whose respective quotients $D$ and $D^{\sigma}$ give conformal mappings of the upper half plane $\mathfrak{h}$ on the open hyperbolic triangles $P Q T$ and $P^{\sigma} Q^{\sigma} T^{\sigma}$ with

$$
\begin{aligned}
D(0) & =P, & D^{\sigma}(0) & =P^{\sigma}, \\
D(\infty) & =Q, & D^{\sigma}(\infty) & =Q^{\sigma}, \\
D(1) & =T, & D^{\sigma}(1) & =T^{\sigma},
\end{aligned}
$$

The function $D^{\sigma} D^{-1}$ gives explicitly the mapping $f$ from the triangle $F_{0}=P Q T$ to the triangle $F_{0}^{\sigma}=P^{\sigma} Q^{\sigma} T^{\sigma}$ whose existence was deduced from the Riemann mapping theorem with boundary in Section 1. Once again the fact that the angles $m \pi \lambda, s \pi \mu, u \pi \nu$ of $F_{0}^{\sigma}$ are integral multiples of the angles $\pi \lambda, \pi \mu$, $\pi \nu$ of $F_{0}$ allows one to complete the construction as in the first method.

3. Construction in terms of abelian varieties. In this construction we deal explicitly with the subtler aspect of exactly which families of abelian varieties are selected by the embedded curve in the theorem.

In particular, we select a complex one-dimensional subfamily of the abelian varietes $\mathfrak{I}_{\mathrm{t}}$ introduced in Section 1 for the interpretation of $\Gamma$ as a modular group. Then $\Delta$ is just the subgroup of $\Gamma$ fixing this subfamily, and the values of the triangle functions introduced in the second construction serve as the coordinates of those $\tau$ describing the subfamily. We first recall a construction carried out in [Wo 3], $\S 2,4$ and 5 . Consider the same hypergeometric differential equation $L$ as in the second construction. A basis of solutions of $L$ can be given as period integrals of a smooth projective model $X(N, z)$ of the curve

$$
y^{N}=x^{A}(1-x)^{B}(1-z x)^{C}
$$

where $N$ is the least common denominator of the rational parameters $a, b$, $c$ and

$$
A=(1-b) N, \quad B=(b+1-c) N, \quad C=a N .
$$

The differential form to be integrated is $d x / y$ which is of the first or second kind if $z \neq 0,1, \infty$, and an eigendifferential for the action $x^{*}$ induced by the automorphism

$$
\chi:(x, y) \mapsto\left(x, \zeta_{N}^{-1} y\right), \quad \zeta_{N}=e^{2 \pi i / N},
$$

of the curve. In fact, we can then choose the $x^{*}$-eigenbasis of $H^{0}(X(N, z), \Omega)$ using differentials of the form $g(x) d x / y^{m}$ where $g$ denotes monomials in $x, 1-x$, $1-z x$, and $m$ lies between 0 and $N-1$. Let $S$ be the subset of these eigendifferentials belonging to eigenvalues which are in the group of primitive $N$ th roots of unity, $(Z / N Z)^{*}$. Let $\alpha$ and $\beta$ be integration cycles on $X(N, z)$ such that all $x^{m}(\alpha), \chi^{m}(\beta), m=0, \ldots, N-1$ generate a subgroup of finite index in $H_{1}(X(N, z), Z)$; this is possible for example by taking Pochhammer cycles around 0 and $1,1 / z$ and $\infty$, respectively [K1]. Then up to isogeny the Jacobian $\operatorname{Jac} X(N, z)$ contains as an essential factor an abelian variety $\mathfrak{T}$ which as a complex torus has the description $C^{\phi(N)} / \Lambda$, where $\phi$ is Euler's function and $\Lambda$ the period lattice of the first kind given by

$$
\Lambda:=\left\{\left(\sigma_{m}(u) \int_{\alpha} w+\sigma_{m}(v) \int_{\beta} w\right)_{w \in S} \mid u, v \in \mathbf{Z}\left[\zeta_{N}\right]\right\}
$$


with embeddings $\sigma_{m}: \boldsymbol{Q}\left(\zeta_{N}\right) \rightarrow \boldsymbol{C}$ sending $\zeta_{N}$ to $\zeta_{N}^{m}$ the $\chi^{*}$-eigenvalue corresponding to $w$. Therefore, $\operatorname{End}_{0} \mathfrak{T} \supset \boldsymbol{Q}\left(\zeta_{N}\right)$ and $\mathfrak{I}$ has generalized complex multiplication type $\sum_{w \in S} \sigma_{m}$. The multiplicity of the eigenvalues $\zeta_{N}^{m}$ is given by

$$
r_{m}=\left\langle\frac{m A}{N}\right\rangle+\left\langle\frac{m B}{N}\right\rangle+\left\langle\frac{m C}{N}\right\rangle-\left\langle\frac{m(A+B+C)}{N}\right\rangle
$$

where $\langle\alpha\rangle$ denotes the fractional part $\alpha-[\alpha]$ of $\alpha \in \boldsymbol{R}$. The possible values of $r_{m}$ are $0,1,2$ and one has $r_{m}+r_{-m}=2$. Since $\sigma_{-m}=\bar{\sigma}_{m}$, the complex multiplication type $\sum_{w \in S} \sigma_{m}$ is in fact of the form

$$
\sum_{j=1}^{d}\left(\xi_{j}+\bar{\xi}_{j}\right)+2 \sum_{j=d+1}^{\phi(N) / 2} \xi_{j}
$$

discussed in Section 1, $d$ being here half the number of one-dimensional eigenspaces of $H^{0}(T, \Omega)$. If $w \in S$ generates such a one-dimensional eigenspace, the quotient $\int_{\beta} w / \int_{\alpha} w$ as a function of $z$ is a hyperbolic triangle function $D_{w}(z)$ and in the two-dimensional case it will be a spherical triangle function. Moreover, if $w$ and $w^{\prime}$ belong to complex conjugate embeddings $\sigma_{m}$ and $\sigma_{-m}$, the $D_{w}(z)$ and $D_{w^{\prime}}(z)$ will be related by fractional linear transformations.

If we replace $\Lambda$ by the homothetic lattice

$$
\left\{\left(\sigma_{m}(u)+\sigma_{m}(v) D_{w}(z)\right)_{w \in S} \mid u, v \in \boldsymbol{Z}\left[\zeta_{N}\right]\right\}
$$

it turns out that the spherical $D_{w}(z)$ are unimportant for the classification of the T's, so our family of abelian varieties will be parametrized by coordinates $\tau=\left(D_{w}(z)\right)_{w \in R}$ where $R$ is a subset of $S$ containing exactly one representative of every pair $w, w^{\prime}$ which corresponds to the multiplicity one embeddings $\sigma_{m}$ and $\sigma_{-m}$. For a suitable choice of $\alpha, \beta$ and $R$ one may always be sure that $\tau$ is in $\mathfrak{h}$, and the monodromy group $\Delta$ will be the subgroup fixing the family $\mathfrak{I}$ constructed here, within the whole modular group for all abelian varieties with endomorphism algebra $\boldsymbol{Q}\left(\zeta_{N}\right)$ and complex multiplication type $\sum_{w \in S} \sigma_{m}$.

As $\mathrm{F}$. Klein $[\mathrm{Kl}, \S 19]$ pointed out, $\Delta$ can be considered as acting on the integration paths of the integral representation of the hypergeometric functions, and so simultaneously on all components of $\tau$, compare for example $\S 5$ of [Wo 4] (the homology group of [Wo 4] $\$ 5.3$ has to be replaced by the fundamental group). In particular, if $\sigma_{m}$ corresponds to $w, \Delta$ acts on $D_{w}(z)$ by the fractional linear transformations comprising $\sigma_{m}(\Delta)$. Since $\Delta$ changes only the basis of the homology and not the curve $X(N, z)$, it is clear that $\Delta$ has the required fixing property.

On completing this construction, $d$ may be a multiple of the number of unramified infinite places of $\boldsymbol{k}$ for $\mathfrak{B}$. This is due to the fact that the totally real field $\boldsymbol{k}$ may be properly contained in $\boldsymbol{Q}(\cos (2 \pi / N))$. For every $w \in R$ there will then be $\phi(N) / 2 n, n=[k: Q]$ different' $w^{\prime} \in R$ such that the corresponding $\sigma_{m}$ agree on $k$. This in turn means that the $\sigma_{m}(\Delta)$ are the same and that the $D_{w^{\prime}}(z)$ in question coincide. With $r=2 n d / \phi(N)$, our family of abelian varieties is therefore essentially parametrized by a complex analytic one-dimensional subset of $\mathrm{b}^{r}$, again fixed by the action of $\Delta$ introduced in Section 1 . In terms of the abelian varieties, the $\mathfrak{I}$ constructed here split up to isogeny into $\phi(N) / 2 n$ isomorphic factors whose endomorphism algebras contain a complex multiplication extension $\boldsymbol{K}$ of $\boldsymbol{k}, \boldsymbol{K} \subset \boldsymbol{Q}\left(\zeta_{N}\right)$, and whose $\phi(N) / 2 n$-fold complex multiplication type is the restriction to $K$ of the complex multiplication type $\sum_{w \in S} \sigma_{m}$ of $\mathfrak{I}$. We can choose a subsystem of $r$ triangle functions $D_{w}(z), w \in R$, such that the corresponding $\sigma_{m}$ are pairwise different on $\boldsymbol{k}$; a look at the action of $\Delta$ on them shows that we get exactly the same $r$ triangle functions as by the 2nd construction.

4. Rationality of the quotient map. The rationality of the quotient map $\psi$ of the Theorem follows immediately from the Proposition stated below, whose proof relies on the following lemma.

Lemma. Let $\Delta$ be a Fuchsian group of finite covolume acting on the unit disc $E_{1}$, and let $g$ be a bounded holomorphic function on $E_{1}$, vanishing on some orbit $\Delta\left(\tau_{0}\right)$. Then $g$ vanishes identically.

Proof. The orbit $\Delta\left(\tau_{0}\right)$ is given by a sequence $\left\{a_{n}\right\}_{n \in N}$ of points in the unit disc with the property that $\sum\left(1-\left|a_{n}\right|\right)$ diverges ([Le], Thm. 2.3.4). Then as a consequence of Jensen's formula, $g\left(a_{n}\right)=0$ for all $n \in N$ implies $g \equiv 0$ (compare [Co], XI § 1, Exercise 5a).

We now show:

Proposition 1. Let $\Delta, \Gamma$ be two discontinuous groups of finite covolume acting respectively on $E_{1}, E_{1}^{r}, r \geqslant 1$, such that the projective varieties given by the compactifications of their quotients, $V_{1}=\overline{E_{1} / \Delta}, V_{2}=\overline{E_{1}^{r} / \Gamma}$ are defined over $\bar{Q}$. Suppose there exists a morphism $p: V_{1} \rightarrow V_{2}$, and a point $v_{1} \in V_{1}(\bar{Q})$ with $p\left(v_{1}\right) \in V_{2}(\bar{Q})$. Then $p$ is defined over $\bar{Q}$.

Proof. Let $U\left(v_{1}\right), U\left(p\left(v_{1}\right)\right)$ be small neighbourhoods of $v_{1}, p\left(v_{1}\right)$ which we lift to neighbourhoods $\widetilde{U}(0), \tilde{U}(0)$ of the origins of $E_{1}, E_{1}^{r}$.

If $p$ is not defined over $\bar{Q}$, we consider small deformations $\tilde{p}$ of $p$ which arise from the action of $\operatorname{Gal}(\boldsymbol{C} / \bar{Q})$ on the coefficients. These leave invariant $V_{2}$ (defined over $\bar{Q}$ ) and all algebraic image points of $p$ in $V_{2}(\bar{Q})$.

The deformed embedded curve $\tilde{p} V_{1} \subset V_{2}$ lifts to an embedded deformed subvariety $\tilde{F} E_{1} \subset E_{1}^{r}$, and by the above choice of lifting and the fact that $v_{1}$, $p\left(v_{1}\right)$ are algebraic, we can assume $\tilde{F}$ is holomorphic and coincides with $F$ on the orbit $\Delta(0)$. Applying the Lemma to the components of $\tilde{F}-F$, we have $\tilde{F} \equiv F$, and so $\tilde{p} \equiv p$.

5. Implications of the modular embedding for the covering radius. We now examine the implications of the Theorem of Section 2 for the original question of S. Lang about covering radii, refered to in the Introduction. 
We begin by returning to the preliminary remarks of Section 1 about the families of abelian varieties associated to certain quaternion algebras. We keep the same notations and for simplicity take $\boldsymbol{k}=\boldsymbol{k}_{0}$.

Now, for any embedding $\varepsilon: K \subset \mathfrak{B}$ there is a common fixed point $\tau_{0} \in \mathfrak{h}^{r}$ of the image, and the member $\mathfrak{I}_{z_{0}}$ for this fixed point $\tau_{0}$ is isogeneous to a sum $V_{1} \oplus V_{2}$ of abelian varieties with complex multiplication in the sense of Shimura and Taniyama, of complex dimension $n$, with endomorphism algebras $\operatorname{End}_{0} V_{i}$, $i=1,2$ containing $\boldsymbol{K}$ and of complex multiplication type

$$
\phi_{1}=\sum_{j=1}^{n} \xi_{j}, \quad \phi_{2}=\sum_{j=1}^{r} \xi_{j}+\sum_{j=r+1}^{n} \xi_{j} .
$$

If we denote the complex multiplication type of such an abelian variety $V$ by $\Sigma=\sum_{j=1}^{n} \sigma_{j}$, all non-zero periods of an algebraically defined eigendifferential $w_{j} \in H^{0}(V, \Omega)$ for $\sigma_{j}$ are algebraic multiples of a positive real number we call $\pi p\left(\sigma_{j}, \Sigma\right)$ (for information on these numbers see in particular [Shi 2]).

By Riemann's period relations, each

$$
\varrho_{j}=: \pi p\left(\xi_{j}, \phi_{1}\right) p\left(\xi_{j}, \phi_{2}\right), \quad j=1, \ldots, r
$$

is a quotient of a period of the first kind by a period of the second kind and is therefore a transcendental number (for a proof of this see [WW]), and by Shimura's period relations ([Shi 2], Thm. 1.2), $\varrho_{j}$ depends only on $\xi_{j}$ and $\phi=\phi_{1}+\phi_{2}$, always up to a factor in $Q^{*}$. A simple application of the results of [Shi 2, Theorems 7.1, 7.6] on derivatives of automorphic functions yields:

PROPOSITION 2. There are generators of the ring of $\Gamma$-automorphic functions on $\mathfrak{b}^{r}$, regular at $\tau_{0}=\left(\tau_{01}, \ldots, \tau_{0 r}\right)$, having series expansions

$$
f(\tau)=\sum_{v_{1}, \ldots, v_{r} \geqslant 0} c_{v_{1} \ldots, v_{r}} \prod_{j=1}^{r}\left(\varrho_{j} \cdot \frac{\tau_{j}-\tau_{0 j}}{\tau_{j}-\bar{\tau}_{0 j}}\right)^{v_{j}}
$$

with algebraic coefficients $c_{v_{1}, \ldots, v_{r}}$.

In particular, we have the following immediate corollary of the above discussion, of the type proposed by $\mathrm{S}$. Lang.

COROLlary. Let $V \cong \overline{E / \Gamma}$ be a Shimura variety, with $\Gamma$ commensurable with the unit group of a maximal order of a quaternion algebra, realized as acting on the product $E=E_{\rho_{1}} \times \ldots \times E_{\rho_{r}}$ of $r \geqslant 1$ discs of radius $\varrho_{j}$ and centre the origin, $j=1, \ldots, r$. Let the corresponding holomorphic (possibly ramified) covering map $\varphi_{V}: E \rightarrow V$ be normalized so that $\varphi_{V}(0)$ is a complex multiplication point, and $\varphi_{V}^{\prime}(0)$ is defined over $\bar{Q}$. Then the radii $\varrho_{1}, \ldots, \varrho_{r}$ are transcendental numbers.

In particular, for $r=1$ we obtain the example of Shimura curves, when $X=V$, mentioned in the Introduction (Case 2). We are now ready to analyse Case 3 of the Introduction. Under the assumptions of the Theorem of Section 2, consider a $\Delta$-fixed point $P \in \mathfrak{h}$ of order $p$. Then $\tau_{0}=F(P)$ is a $\Gamma$-fixed point of order $p$, and so $\boldsymbol{k} \cdot 1$ and $\gamma_{P}$ generate a subfield $\boldsymbol{K} \cong \boldsymbol{k}\left(\zeta_{p}\right) \subset \mathfrak{B}$ fixing $\tau_{0}$. In this way, $\tau_{0}$ is a complex multiplication point to which the above corollary applies. If we choose the covering domains of $X$ and $V$ so that $P, \tau_{0}$ are represented at the origin with the modular embedding now written $F: E_{Q} \rightarrow E$ $=E_{e_{1}} \times \ldots \times E_{e_{r}}$ for the $\varrho, \varrho_{j} ; j=1, \ldots, r$ required for the condition that $d_{\varphi}$, $d_{\varphi v}$ be algebraic at $0, \underline{0}$, then the $\varrho_{j}, j=1, \ldots, r$ are determined by the preceding discussion and we have a commutative diagram:

$$
\begin{aligned}
& E_{Q} \stackrel{F}{\rightarrow} E=E_{Q_{1}} \times \ldots \times E_{e_{r}} \\
& \downarrow_{\varphi} \quad \downarrow_{\varphi V} \\
& X \longrightarrow V
\end{aligned}
$$

Moreover, $d \psi$ is algebraic at $\varphi(0)$ by the Theorem, and therefore $d F$ is defined over $\boldsymbol{Q}$ at $0 \in E_{\rho}$. But, in the course of the proof of the Theorem, we saw that the first component of the modular embedding written in terms of $h$ could be taken as the identity, so that in the present representation the first component of $F$ is $\left(\varrho_{1} / \varrho\right) \cdot 1_{E_{e}}$. By the commutativity of the above diagram, $\varrho$ and $\varrho_{1}$ must coincide up to an algebraic factor, so that in particular $\varrho$ is transcendental. The preceding discussion of the nature of the $\varrho_{j}, j=1, \ldots, r$ provides the elucidation of Case 3 promised in the Introduction. (See also [WW].)

One knows that the above radii $\varrho_{j}, j=1, \ldots, r$ must be expressible in terms of beta-functions at rational arguments. We briefly record the relevant formulae here, although they already appear implicitly in [WW] for $j=1$, and for $j=1, \ldots, r$ in [Wo 3].

Using $\sim$ to denote the equivalence of two real numbers up to multiplication by a non-zero algebraic number, it follows for example from [WW, p. 11] that with the origin of the covering disc a fixed point of order $p$, the condition that the derivative of the covering map at the origin be algebraic forces the disc to have radius:

$$
\begin{aligned}
& \varrho \sim \varrho_{1} \sim \frac{B\left(\frac{1}{p}, \frac{1}{2}\left(1-\frac{1}{p}+\frac{1}{q}-\frac{1}{t}\right)\right)}{B\left(1-\frac{1}{p}, \frac{1}{2}\left(1+\frac{1}{p}+\frac{1}{q}+\frac{1}{t}\right)\right)} \\
& \sim \frac{1}{\pi} B\left(\frac{1}{p}, \frac{1}{2}\left(1-\frac{1}{p}+\frac{1}{q}-\frac{1}{t}\right)\right) B\left(\frac{1}{p}, \frac{1}{2}\left(1-\frac{1}{p}-\frac{1}{q}-\frac{1}{t}\right)\right) .
\end{aligned}
$$

By the discussion of $\S 3.2$, the remaining radii $\varrho_{j}, j=2, \ldots, r$ can be given in an analogous way if $1 / p, 1 / q, 1 / t$ are replaced by appropriate multiples $m / p$, $s / q, u / t$.

In fact with $N=$ l.c.m. $(p, q, t)$ and $A, B, C$ as in $\S 3.3$ there is an $m \in(Z / N Z)^{*}$ with (using also [Wo 3], \& 6) 


$$
\begin{gathered}
r_{m}=\left\langle\frac{m A}{N}\right\rangle+\left\langle\frac{m B}{N}\right\rangle+\left\langle\frac{m C}{N}\right\rangle-\left\langle\frac{m(A+B+C)}{N}\right\rangle=1, \\
\left\langle m \frac{A}{N}\right\rangle+\left\langle m \frac{B}{N}\right\rangle>1
\end{gathered}
$$

such that

(10) $\varrho_{j} \sim \frac{1}{\pi} B\left(\left\langle m \frac{A+B}{N}\right\rangle,\left\langle-m \frac{A}{N}\right\rangle\right) B\left(\left\langle m \frac{A+B}{N}\right\rangle,\left\langle m \frac{C}{N}\right\rangle\right), \quad j=2, \ldots, r$ and that with $j=m=1$, we recapture (9). For a more detailed discussion the reader is advised to compare Section 3.3 with [Wo 3].

We remark in passing that classical expressions for the series expansions of triangle functions about elliptic fixed points (as in [Car], $\S 392$, p. 145) in terms of hypergeometric functions (or their reexpression in terms of beta-functions as above) with rational parameters, show on inspection that the radii $\varrho_{j}$ are exactly those for which the function $D_{e}$, now defined as mapping the unit disc into the interior of a hyperbolic triangle with angles $m \lambda, s \mu$, $\dot{u} v$ inside $E_{e}$, has a power series around the origin with rational coefficients, a property shared by the function $f_{j}: D_{e_{j}} \circ D_{e_{1}}^{-1}: E_{e_{1}} \rightarrow E_{e^{\prime}}, j=1, \ldots, r$. With this type of observation for example, one can furnish another proof of the rationality of the map $\psi$ of the Theorem.

We end this section by recalling that in $[W W, \S 4]$ it is observed that for triangle groups $\Delta$ with cusps, corresponding to pointed curves $X$, the covering radii found there can always be written as a square of a period of the first kind divided by $\pi$. Here, $\Delta$, and therefore the algebra $\mathfrak{B}$ has unipotent elements, so $\mathfrak{B} \otimes_{\mathbb{Q}} \boldsymbol{R}$ cannot have factors isomorphic to the Hamiltonians. The complex multiplication types in question must be of the form $\phi_{1}, \phi_{2}=\bar{\phi}_{1}$, and Shimura's derivatives become:

$$
\varrho_{j} \sim \pi p\left(\xi_{j}, \phi_{1}\right) p\left(\xi_{j}, \bar{\phi}_{1}\right) \sim \pi p\left(\xi_{j}, \phi_{1}\right)^{2}, \quad j=1, \ldots, r .
$$

A. Selberg has shown that in this case $\Gamma$ is commensurable to the Hilbert modular group belonging to $\boldsymbol{k}$. Here the family of abelian varieties can be simplified considerably. All $\mathfrak{T}_{\text {, }}$ not only those at the complex multiplication points, are isogeneous to a product of two isomorphic factors with real multiplication by $\boldsymbol{k}$, and these factors can serve as members of a new family again with modular group $\Gamma$.

\section{Examples}

1. Let the signature $(p, q, t)$ be $(5, \infty, \infty)$. Up to conjugation, $\Delta$ is generated by the parabolic matrices

$$
\gamma_{Q}=\left[\begin{array}{ll}
1 & 1 \\
0 & 1
\end{array}\right] \text { and } \gamma_{T}=\left[\begin{array}{ll}
1 & 0 \\
\alpha & 1
\end{array}\right]
$$

with $\alpha=(-3+\sqrt{5}) / 2$. So $Q=\infty, T=0$, and $P$ will be a fixed point of order 5 of

$$
\gamma_{P}= \pm\left(\gamma_{Q} \gamma_{T}\right)^{-1}
$$

The group $\Delta$ is non-arithmetic [Ta 1$]$ and is contained in the Hilbert modular group of the real quadratic field $\boldsymbol{k}=\boldsymbol{Q}(\sqrt{5})$. The algebraic conjugation $\sigma: \alpha \mapsto \bar{\alpha}$ of $k$ induces a group homomorphism $\Delta \rightarrow \Delta^{\sigma}$, where the image $\Delta^{\sigma}$ is generated by $\gamma_{Q}^{\sigma}=\gamma_{Q}$ and $\gamma_{T}^{\sigma}=\left[\begin{array}{ll}1 & 0 \\ \bar{\alpha} & 1\end{array}\right]$.

In the construction of the modular embedding $\tau \mapsto(\tau, f(\tau)) \in \mathrm{h}^{2}$ we have to find a function $f$ analytic on $\mathfrak{h}$, satisfying

$$
f(\gamma \tau)=\gamma^{\sigma} f(\tau) \quad \text { for all } \tau \in \mathfrak{h}, \gamma \in \Delta .
$$

This will be given by the analytic continuation of the conformal mapping of the left half of the fundamental domain for $\Delta$ onto the hyperbolic triangle $P^{\sigma} Q^{\sigma} T^{\sigma}$ with angles $(3 \pi / 5,0,0)$. According to Section $2, f=D_{2} \circ D_{1}^{-1}$ where $D_{1}, D_{2}$ are the respective triangle functions for the angles $(\pi / 5,0,0),(3 \pi / 5,0,0)$. The corresponding parameters for the hypergeometric differential equation with monodromy group $\Delta$ are $(a, b, c)=(2 / 5,2 / 5,4 / 5)$, and the curve involved in the construction via abelian varieties is a smooth projective model $X(5, z)$ of

$$
y^{5}=x^{3}(1-x)^{3}(1-z x)^{2} .
$$

As expected by the remarks on triangle groups with cusps (Section 5), the dimensions of the $\boldsymbol{Q}\left(\zeta_{5}\right)$-eigenspaces of our family of abelian varieties, given by the Jacobians of $X(5, z)$ are

$$
r_{m}=\left\langle m \frac{3}{5}\right\rangle+\left\langle m \frac{3}{5}\right\rangle+\left\langle m \frac{2}{5}\right\rangle-\left\langle m \frac{8}{5}\right\rangle=1
$$

for all $m \in(\mathbf{Z} / 5 \mathbf{Z})^{*}$.

By our remarks of Section 5 for the identification of which $D_{j}$ occur in the construction we have to choose such $m \in(Z / 5 Z)^{*}$ with $r_{m}=1$, always true in this case, and $\left\langle m \frac{A}{N}\right\rangle+\left\langle m \frac{B}{N}\right\rangle>1$, i.e. $2\left\langle m \frac{3}{5}\right\rangle>1$, so $m=1,3$ and we recover again the angle triples $(\pi / 5,0,0),(3 \pi / 5,0,0)$. The radius of the covering disc with origin a fixed point of $\Delta$ of order 5 , for $X=\Delta \backslash \mathfrak{h}$ is $\varrho \sim \frac{1}{\pi} B(1 / 5,2 / 5)^{2}$, and the covering radii of the Shimura variety $V$ with origin the corresponding points are given by (10),

$$
\varrho_{1} \sim \frac{1}{\pi} B(1 / 5,2 / 5)^{2} \quad \text { and } \quad \varrho_{2} \sim \frac{1}{\pi} B(3 / 5,1 / 5)^{2} .
$$


The beta-values are the periods of algebraically defined differentials of the first kind on a simple abelian variety of complex dimension 2, complex multiplication by $\boldsymbol{Q}\left(\zeta_{5}\right)$ and complex multiplication type $\{1,3\}$ (this notation is explained in [Wo 3], and here corresponds to the embeddings mapping $\zeta_{5} \mapsto \zeta_{5}$, $\left.\zeta_{5} \mapsto \zeta_{5}^{3}\right)$.

The above triangle group is not maximal; it is contained in the Hecke group of index 10 , i.e. the triangle group of signature $(10,2, \infty)$. Here for the fixed points of order 10 , one obtains again the range $\{1,3\}$ for $m$ and, using classical relations for beta functions, the same covering radii. The modular embedding is more complicated than the previous one as the field $Q(\cos (\pi / 10))$ is of degree 4; the abelian varieties are $\operatorname{Jac} X(10, z), X(10, z): y^{10}$ $=x^{3}(1-x)^{8}(1-z x)^{2}$. Once again $\Delta$ is embeddable in the Hilbert modular group of $Q(\sqrt{5})$.

Now consider the non-arithmetic Hecke group $\Delta$ of signature $(5,2, \infty)$. Here again $k=Q(\cos (\pi / 10))$, and what is more the modular embedding of $\Delta$ in the Hilbert modular group of $\boldsymbol{k}$ can be reduced to a simpler modular embedding, where $\boldsymbol{k}$ is replaced by $\boldsymbol{Q}(\sqrt{5})$. This time one has:

$$
\begin{gathered}
(a, b, c)=(3 / 20,13 / 20,4 / 5), \\
X(20, z): y^{20}=x^{7}(1-x)^{17}(1-z x)^{3}, \\
m \in\{1,-3,7,-9\} \subset(Z / 20 Z)^{*}
\end{gathered}
$$

giving rise (by a simple application of [Wo 3], (16), (17)) to four triangle functions, but only two distinct ones. Therefore the image $F(b)$ of the modular embedding lies in a 2-dimensional symmetric subdomain of $b^{4}$. This subdomain parametrizes those 4-dimensional abelian varieties $\mathfrak{I}$ with $\operatorname{End}_{0}(\mathfrak{I}) \supseteq \boldsymbol{Q}\left(\zeta_{20}\right)$ which split into two isomorphic factors $\mathfrak{T}_{1}$ and $\mathfrak{I}_{2}$, both with $\operatorname{End}_{0} \mathfrak{I}_{i} \supseteq \boldsymbol{Q}\left(\zeta_{5}\right)$, $i=1,2$. On this 2-dimensional subvariety, the modular group is the Hilbert modular group of $Q(\sqrt{5})$. Notice that the subgroup $\{1,-9\}$ of $(Z / 20 Z)^{*}$ $\cong \operatorname{Gal}\left(Q\left(\zeta_{20}\right) / Q\right)$ is the fixing subgroup of $Q\left(\zeta_{5}\right)$, and stabilizes the C-M type $\{1,-3,7,-9\}$, the orbit corresponding to the coinciding triangle functions. Of course, one obtains exactly the same covering radii as for $(5, \infty, \infty)$ with double multiplicity.

It would be interesting to know if the curves $\psi(X)$ defined by these modular embeddings on the Hilbert modular surfaces $V$ for $Q(\sqrt{5})$ are linearly equivalent to Hirzebruch-Zagier cycles.

2. For our second example, we mention a class of compact curves $X$ to which our modular embedding procedure applies: The Fermat curves of exponent $p>3$ are quotients of $\mathfrak{h}$ by the commutator subgroup of a triangle group $\Delta$ with signature $(p, p, p)$. According to Takeuchi $[$ Ta 1,2$], \Delta$ is arithmetic so that the Fermat curve is a Shimura curve, exactly for $p=4,5,6$, $7,8,9,12,15$. The first nontrivial $p=10$ leads to a two-dimensional Shimura variety $V$. For $p$ even one obtains $N=2 p, A=p+1, B=p+1, C=p-3$, and for $p=10$ one has $r_{m}=1$ exactly when $\pm m=1$ or 3 . All the other $m \in(\boldsymbol{Z} / 20 \boldsymbol{Z})^{*}$ satisfy $r_{m}=0$ or 2 , so correspond to embeddings of $\Delta$ in the hamiltonians. To $m=1$ and 3 correspond the angles $\pi / 10, \pi / 10, \pi / 10$ and $3 \pi / 10,3 \pi / 10,3 \pi / 10$. The covering radii of $X$ and $V$ with point of order 10 at the origin are

$$
\varrho \sim \varrho_{1} \sim \frac{1}{\pi} B(1 / 10,9 / 20) B(1 / 10,7 / 20)
$$

and

$$
\varrho_{2} \sim \frac{1}{\pi} B(3 / 10,7 / 20) B(3 / 10,1 / 20) .
$$

The first beta-factors are periods on the abelian variety $W_{1}$ of complex multiplication type $\psi_{1}=\{1,3,7,9\}$ and the second beta-factors are periods on the abelian variety $W_{2}$ of complex multiplication type $\overline{\psi_{2}}=\{1,3,-7,-9)$, both for the field $Q\left(\zeta_{20}\right), r_{m}$ of course being the multiplicity of $m$ in $\psi_{1}+\psi_{2}$.

At the end of this section we shall make some remarks about improving the non-injectivity of the morphism $\psi: X \rightarrow V$ of Fermat curves into Shimura varieties.

3. Sometimes, there are modular embeddings

$$
F=\left(f_{1}, \ldots, f_{r}\right): \mathfrak{h} \rightarrow \mathfrak{h}^{r}
$$

associated to homomorphisms $h: \Delta \hookrightarrow \Gamma$ so that

$$
F(\gamma \tau)=h(\gamma) F(\tau) \quad \text { all } \tau \in \mathfrak{h}, \gamma \in \Delta
$$

where $h$ is not given by the natural embeddings of Section 3 nor by obvious combinations invoking permutations of the coordinates of $b^{r}$. These new $F$ may lack the identity component of the natural embeddings, and may become singular in the elliptic fixed points.

More specifically, consider a homomorphism $h$ obtained by blowing up the angles in $P, Q, T$ by factors $m, s, u$ not coming from an algebraic embedding $\boldsymbol{k} \subset \boldsymbol{R}$, in such a way that one preserves the group relations. Then one has the same principle of construction for $F$ as in the proof of the Theorem.

Take, for example, the triangle group of signature $(10,10,10)$ of example 2 above. Choose a hyberbolic triangle $P^{\prime}, Q^{\prime}, T^{\prime}$ within $\mathfrak{h}$ with angles $3 \pi / 10$, $\pi / 10, \pi / 10$ such that their fixing transformations $\gamma_{P^{\prime}}, \gamma_{Q^{\prime}}, \gamma_{T^{\prime}}$ satisfy

$$
\operatorname{tr} \gamma_{P^{\prime}}= \pm 2 \cos (3 \pi / 10), \quad \operatorname{tr} \gamma_{Q^{\prime}}= \pm 2 \cos (\pi / 10), \quad \operatorname{tr} \gamma_{T^{\prime}}= \pm 2 \cos (\pi / 10),
$$

$$
\gamma_{\mathbf{P}^{\prime}} \gamma_{Q^{\prime}} \gamma_{T^{\prime}}=\gamma_{P} \gamma_{Q} \gamma_{T}= \pm 1
$$

and $\gamma_{\boldsymbol{P}^{\prime}}, \gamma_{Q^{\prime}}, \gamma_{T^{\prime}} \in \mathrm{SL}_{2}(\boldsymbol{Q}(\cos (\pi / 10)))$. Again, these generate a quaternion algebra $\mathfrak{B}$ over $\boldsymbol{Q}(\cos (\pi / 10))$, different from the one used in example 2 , and are contained in a group $\Gamma$ of units of reduced norm 1 in some order of $\mathfrak{B}$. 
Defining

$$
h\left(\gamma_{P}\right)=\gamma_{P^{\prime}}, \quad h\left(\gamma_{Q}\right)=\gamma_{Q^{\prime}}, \quad h\left(\gamma_{T}\right)=\gamma_{T^{\prime}}
$$

one has an injective homomorphism $h: \Delta \hookrightarrow \Gamma$. It turns out that $r=3, n=4$, and that the three embeddings of $\mathfrak{B}$ into $M_{2}(R)$ which describe the action of $\Gamma$ on $\mathfrak{h}^{3}$ extend the embeddings of $\boldsymbol{Q}(\cos (\pi / 10))$ into $\boldsymbol{R}$ with, respectively, $\cos (\pi / 10) \mapsto \cos (\pi / 10), \cos (7 \pi / 10), \cos (9 \pi / 10)$.

One way to calculate these is to use (5) and (6) for the determination of the curve

$$
X(N, z): y^{20}=x^{13}(1-x)^{13}(1-z x)^{5},
$$

so that $r_{m}=1$ exactly for $\pm m=1,7,9$.

One can continue to find the explicit shape of the components $f_{1}, f_{2}, f_{3}$ of the desired $F$ as in Section 3: they are composed of the inverse of the triangle function with angles $\pi / 10, \pi / 10, \pi / 10$, and of triangle functions with angle triples $(3 \pi / 10, \pi / 10, \pi / 10),(\pi / 10,-3 \pi / 10,-3 \pi / 10)$ and $(7 \pi / 10,-\pi / 10,-\pi / 10)$.

Of course, this type of modular embedding is not interesting for information on the covering radius of $X$, but perhaps for the construction of some more algebraic cycles on $V$.

We end this section by reconsidering the morphism $\psi: X \rightarrow V$ of Fermat curves into Shimura varieties as advertised in example 2 .

Let $\Delta$ be of signature $(p, p, p)$ and consider the modular embedding $F$ constructed in the Theorem. As pointed out in Section 3 the question if the quotient map is an immersion may be reformulated as follows: does there exist a subgroup $\Gamma^{\prime}$ of finite index in $\Gamma$ such that the commutator subgroup $[\Delta, \Delta]$, which is the fundamental group of the Fermat curve, is the fixing subgroup of $F(\mathfrak{h})$ :

$$
\left\{\gamma \in \Gamma^{\prime} \mid \gamma F(\mathfrak{h})=F(\mathfrak{h})\right\} ?
$$

For most congruence subgroups $\Gamma^{\prime}$ of $\Gamma$, this is not the case, since by results of Macbeath [Ma] that for all prime ideals $\mathfrak{I}$ in the rationality field of $\Delta$ which do not divide $p$, the projective group $\Delta \bmod \mathfrak{T}$ is all of $\operatorname{PSL}_{2}(F)$ where $\boldsymbol{F}$ is the finite residue class field, so that $[\Delta, \Delta] \bmod \mathfrak{I}$ is no smaller than $\Delta \bmod \mathfrak{T}$.

In the case where $p$ is even, for example, one can get a better result with the ideas developed in example 3 , if one chooses not the identical embedding of $\Delta$ into a modular group, but rather one which doubles exactly one angle of the three original angles $\pi / p$. Then using Macbeath's singular triples one can construct a much finer $\Gamma^{\prime}$, in the sense that $F$ will then induce a 2-to-1 quotient map $\psi$ of the Fermat curve onto a curve in $\Gamma^{\prime} / h^{r}$.

7. Concluding remarks. It is an open question whether our methods and results apply to a larger class of Fuchsian groups $\Delta$ of finite covolume in $\mathfrak{h}$, and contained in arithmetically defined groups $\Gamma$ acting on $\mathfrak{h}^{r}$, at least if $\Delta$ is commensurable to a group with an elliptic fixed point, which would be a candidate for a complex multiplication point. On the one hand for any group homomorphism: $\Delta \rightarrow \Delta_{j}: \gamma \mapsto \gamma_{j}$, it is possible to find functions $f_{j}: \mathfrak{h} \rightarrow \boldsymbol{C}$ such that $f_{j}(\gamma \tau)=\gamma_{j} f_{j}(\tau)$ for all $\tau \in \mathfrak{h}, \gamma \in \Delta$. For instance, one may take suitable "fonctions zéta-fuchsiennes" in the sense of Poincaré, or quotients of two linearly independent solutions of the Riemann-Hilbert problem for the homomorphism of the covering group of a Riemann surface onto any other group $\Delta_{j} \subseteq \mathrm{GL}_{2}(C)$ which serves as a realization of the monodromy group. On the other hand, it appears quite difficult, and as yet unclear to us, to settle the problem of constructing the $f_{j}$ in such a way that $f_{j}(\mathfrak{h})=\mathfrak{h}$. In particular, it is not possible for arbitrary homomorphisms $\Delta \rightarrow \Delta_{j}$ and it is not possible to find such $f_{j}$ in the case where $\Delta_{j}$ is a Fuchsian group of the same signature as $\Delta$, but giving a Riemann surface $\Delta \backslash \mathfrak{h}$ non-isomorphic to $\Delta \backslash \mathfrak{h}$, as there would then be a nontrivial quotient map between them. It is clear therefore that some more arithmetic properties have to be invoked such as the fact that $\Delta_{j}$ is related by Galois conjugation to $\Delta$, or the existence of elliptic fixed points whose images are determined by the homomorphism $\Delta \rightarrow \Delta_{j}$ and which in turn determine the $f_{j}$ uniquely, using arguments of the sort in the Lemma of Section 4.

The aspects of modular embeddings presented in this article have been specifically motivated by considerations from transcendence theory and techniques from quaternion algebras which are necessarily Galoisian in nature.

A quite different problem has been tackled by $\mathrm{Kra}[\mathrm{Kra}]$ who is interested in locally homeomorphic maps of the unit disc onto a subset of the complex sphere compatible with a homomorphism of a Fuchsian group acting on the unit disc to a group of Möbius transformations.

Clearly, the maps corresponding to the homomorphisms we consider are not locally homeomorphic, in particular not at the elliptic fixed points. The work of Kra shows that this fact corresponds to the non-discreteness of the Galois conjugates $\Delta^{\sigma}$ of our Fuchsian group $\Delta$.

\section{References}

[A] A. A. Albert, A solution of the principal problem of Riemann matrices, Ann. Math. 35 (1934), 500-515.

[Car] C. Carathéodory, Funktionentheorie, II, Birkhäuser Verlag, Basel, Stuttgart 1961.

[Co] J. B. Conway, Functions of One Complex Variable, 2nd ed., GTM 11, Springer, New York-Heidelberg-Berlin 1978.

[Fr] E. Freitag, Fortsetzung von automorphen Funktionen, Math. Ann. 177 (1968), 95-100.

[Ha] W. F. H a mmond, The modular groups of Hilbert and Siegel, Amer. J. Math. 88 (1966) 497-516.

[KI] F. Klein, Vorlesungen über die hypergeometrische Funktion, Springer Grundlehren 39, Berlin-Heidelberg-New York 1981

[Kli] H. Klingen, Über einen Zusammenhang zwischen Siegelschen und Hermiteschen Modulfunktionen, Abh. Math. Sem. Univ. Hamburg 27 (1964), 1-12.

[Kra] I. K ra, Deformations of Fuchsian Groups, Duke Math. Journ. 36 (1969), 537-546.

- Acta Arithmetica 56 
[La] S. Lang, Introduction to Transcendental Numbers, Addison-Wesley, New York 1966.

[Le] J. Lang, Introduction to Transcendentans (W. J. Harvey ed.), Academic Press, London-New York-San Francisco 1977, pp. 73-120.

[Ma] A. M. M acbeath, Generators of the Linear Fractional Groups, Proc. Amer. Math. Soc. 12 (1968), 14-32.

[Shi 1] G. Shimu ra, On analytic families of polarized abelian varieties and automorphic functions, Ann. Math. 78 (1967), 149-192.

[Shi 2] - Automorphic forms and the periods of abelian varieties, J. Math. Soc. Japan 31 (1979), 561-592.

[Sie 1] C. L. Siegel, Lectures on Riemann Matrices, Tata Institute, Bombay 1963.

[Sie 2] - Bestimmung der elliptischen Modulfunktion durch eine Transformationsgleichung, Abh. Math. Sem. Hamburg 27 (1964), 32-38.

[Ta 1] K. Takeuchi, Arithmetic triangle groups, J. Math. Soc. Japan 29 (1977), 91-106.

[Ta 2] - Commensurability classes of arithmetic triangle groups, J. Fac. Sci. Univ. Tokyo, Sec. I A, 24 (1977), 201-212.

[Wo1] J. Wolfart, Eine arithmetische Eigenschaft automorpher Formen zu gewissen nicht-arithmetischen Gruppen, Math. Ann. 262 (1983), 1-21.

[Wo2] - Taylorentwicklungen automorpher Formen und ein Transzendenzproblem aus der Uniformisierungstheorie, Abh. Math. Sem. Univ. Hamburg 54 (1984), 25-33.

[Wo 3] - Werte hypergeometrischer Funktionen, Invent. Math 92 (1988), 187-216.

[Wo 4] - Fonctions hypergéométriques, arguments exceptionnels et groupes de monodromie, Publ. Math. de I'Université P. et M. Curie, Problèmes Diophantiens 1985-1986, IX.1-IX.24.

[WW] J. Wolfart und G. Wüstholz, Der Überlagerungsradius gewisser algebraischer Kurven und die Werte der Betafunktion an rationalen Stellen, Math. Ann. 273 (1985), 1-15.

LABORATOIRE DE MATH. FONDAMENTALES

UER 48. UNIVERSITÉ P. ET M. CURIE

4 place Jussien

F.75230 Paris Códex 05

France

FACHBEREICH MATHEMATIK

UNIVERSTTAT FRANKFURT

Robert-Mayer-Strasse $6-10$

Dobert-Mayer-Strasse 6-10

\section{Mesures de transcendance pour les quotients de périodes d'intégrales elliptiques}

par

NORIKo HiRATA-KoHNO* (Nara)

1. Introduction et résultats. Soient $\wp, \wp^{*}$ deux fonctions elliptiques de Weierstrass d'invariants algébriques, associées à deux réseaux $\Omega, \Omega^{*}$ de $C$ respectivement. Soit $\mathcal{O}$ l'anneau d'endomorphismes de $\wp$, c'est-à-dire l'anneau des nombres complexes $\varrho$ tels que $\varrho \Omega$ soit contenu dans $\Omega$. On sait que $\mathcal{O}$ est ou bien l'anneau des entiers rationnels $\boldsymbol{Z}$, ou bien un sous-anneau d'indice fini de l'anneau des entiers algébriques d'un corps quadratique imaginaire $k$. Si $\mathcal{O} \neq \boldsymbol{Z}$, on dit que $\wp$ admet des multiplications complexes. Soient $\omega \in \Omega, \omega^{*} \in \Omega^{*}$, des périodes non nulles de $\wp$ et de $\wp^{*}$ respectivement, et $\omega_{1}, \omega_{2} \in \Omega$ deux périodes de $\wp$, linéairement indépendantes sur le corps des nombres réels $R$.

Dans ce papier, on donne des minorations des formes linéaires à coefficients algébriques, ou bien de deux périodes de $\wp$, ou bien d'une période de $\wp$ et de $2 \pi i$, ou bien d'une période de $\wp$ et d'une période de $\wp^{*}$. Ces minorations améliorent les résultats antérieurs. Une minoration de formes linéaires beaucoup plus générales de points algébriques de l'application exponentielle de groupes algébriques commutatifs sera traitée dans un autre article de l'auteur [H 2], qui permettra de raffiner l'estimation de P. Philippon et M. Waldschmidt [P-W 1].

1.1. Les deux théorèmes principaux. Pour énoncer les résultats précis, rappelons la définition de la hauteur $h$ de Weil logarithmique absolue: pour $\alpha=\left(\alpha_{0}, \ldots, \alpha_{N}\right) \in \boldsymbol{P}_{N}(\overline{\boldsymbol{Q}})$, si $K$ est un corps de nombres contenant $\alpha_{0}, \ldots, \alpha_{N}$, on définit $h$ par

$$
h(\alpha)=\frac{1}{[K: \boldsymbol{Q}]} \sum_{v}\left[K_{v}: \boldsymbol{Q}_{v}\right] \log \operatorname{Max}\left\{\left|\alpha_{j}\right|_{v} ; 0 \leqslant j \leqslant N\right\}
$$

où $v$ décrit l'ensemble des places de $K$ et $\left[K_{v}: Q_{v}\right]$ est le degré local de telle sorte que la formule du produit s'écrive pour $\gamma \in K, \gamma \neq 0$,

$$
\sum_{v}\left[K_{v}: Q_{v}\right] \log |\gamma|_{v}=0 .
$$

* Ces recherches sont subventionnées par une bourse du gouvernement français. 